\title{
The Value of OCT GCIPL Parameters in Neuro-Ophthalmology- A Review Article
}

\begin{abstract}
Ahmed Darwish*
Professor of Ophthalmology, Ain Shams University, Egypt and Vitreoretinal Surgery

Consultant at Dhahran Eye Specialist Hospital, KSA

*Corresponding Author: Ahmed Darwish, Professor of Ophthalmology, Ain Shams University, Egypt and Vitreoretinal Surgery Consultant at Dhahran Eye Specialist Hospital, KSA.
\end{abstract}

Received: August 27, 2021

Published: October 04, 2021

(C) All rights are reserved by Ahmed Darwish.

\begin{abstract}
Purpose: Currently, segmentation of individual retinal layer is possible using spectral-domain OCT (SD-OCT). The Ganglion Cell/ Inner plexiform layer (GCIPL) investigation has become one of the most useful tools in neuro-ophthalmic practice. In this review we shall point out the value of this investigative tool in neuro-ophthalmology.

Methods: To evaluate the above-mentioned item based on recently published data.

Results: GCIPL analysis may provide earlier information about neuronal damage than retinal nerve fiber layer (RNFL) analysis in neuro-ophthalmic disorders.

Conclusion: Optical coherence tomography GCIPL testing has proved to be an important non invasive less time consuming test in the diagnosis, treatment, and follow-up of optic neuropathies.
\end{abstract}

Keywords: Neuro-Ophthalmology; SD OCT; Optical Coherence Tomography; Ganglion Cell Inner Plexiform Layer

\section{Introduction}

Recent advances in OCT segmentation technology allows the thickness of individual layers of the retina to be quantified. This, in turn, can facilitate quantification of axonal loss (RNFL thinning) and neuronal damage [ganglion cell inner plexiform layer (GCIP) thinning] by various mechanisms causing damage to the optic nerve, chiasm, and optic tract [1].

OCT measured thinning of the macular GCIP has been found to correlate strongly with visual loss in a wide spectrum of optic neuropathies including glaucoma, idiopathic intracranial hypertension (IIH) optic neuritis (ON), ischemic optic neuropathy, he- reditary optic neuropathy, toxic optic neuropathy, and tumors of the afferent visual pathway [2].

\section{Idiopathic intracranial hypertension (IIH)}

OCT GCIL layer measurements are used to monitor the integrity of the optic nerve in the setting of papilledema.

Following the peripapillary RNFL thickness over follow up visits may provide an indication of changes in the papilledema.

However, a reduction in peripapillary RNFL thickness in papilledema can be either a result of improving papilledema or progressive axonal loss from inadequate or improper treatment. 
Combining the macular GCIP thickness with the peripapillary RNFL thickness allows one to evaluate the true degree of axonal damage in the setting of papilledema [3].

Successful treatment with preservation of axonal integrity will be identified on OCT as simultaneous reduction in the peripapillary RNFL thickness with a preserved macular GCIP thickness. However, a reduction in the RNFL thickness and macular GCIP thickness simultaneously indicates progressive axonal damage and could be an indication of improper or treatment failure for IIH [4].

Errors in segmentation of the GCIP in the setting of high degrees of papilledema can lead to a falsely thin GCIP thickness giving a false alarm of worsening disease if they are not recognized.

These artifacts can however, be identified because they result in significant reductions in the GCIP thickness in an unusual pattern [2].

\section{Central nervous system (CNS) demyelinating disease}

OCT GCIP layer thickness is not needed to diagnose optic neuritis (ON), but it, however, may offer some prognostic information. OCT measured RNFL values are initially elevated in acute $\mathrm{ON}$, due to axoplasmic flow stasis secondary to inflammation of the optic nerve. This fact makes it difficult to rely on RNFL thickness to monitor axonal integrity because it can often take 2 - 3 months for RNFL thinning to manifest after $\mathrm{ON}$.

On the contrary, however, OCT measured GCIP changes represent an early, sensitive, and reliable sign of retrobulbar neuroaxonal injury. It can be a true indicator for the quantification of neuronal damage in the setting of demyelination of the optic nerve, chiasm, and optic tract [1].

A reduction in post acute OCT GCIP measures correlate with reduced high and low contrast letter acuity, color vision, and visual field sensitivity testing in ON eyes [5].

\section{OCT GCIP value in identifying the underlying etiology}

Nearly all causes of ON lead to thinning of the GCIP layer, yet the amount of loss can be helpful in identifying the underlying etiology.

Several studies have shown that optic neuritis eyes in neuromyelitis optica spectrum disorders (NMOSD) patients have more pronounced thinning of the macular GCIP when compared to MS ON eyes [6].

OCT GCPI for localization and follow up of MS lesions at any part of the afferent visual pathway

Lesions in the afferent visual system of MS patients are not limited to the optic nerve, but may also affect the afferent visual pathway back to the retrogeniculate regions.

MS patients with retrochiasmatic lesions may report symptoms consistent with homonymous visual field defects. A pattern of hemiretinal GCIP thinning that corresponds to the hemianopic visual field defect is usually present moreover, in cases of relapsing disease, changes in GCIPL thickness persist even after visual function has recovered [8] (Figure 1).

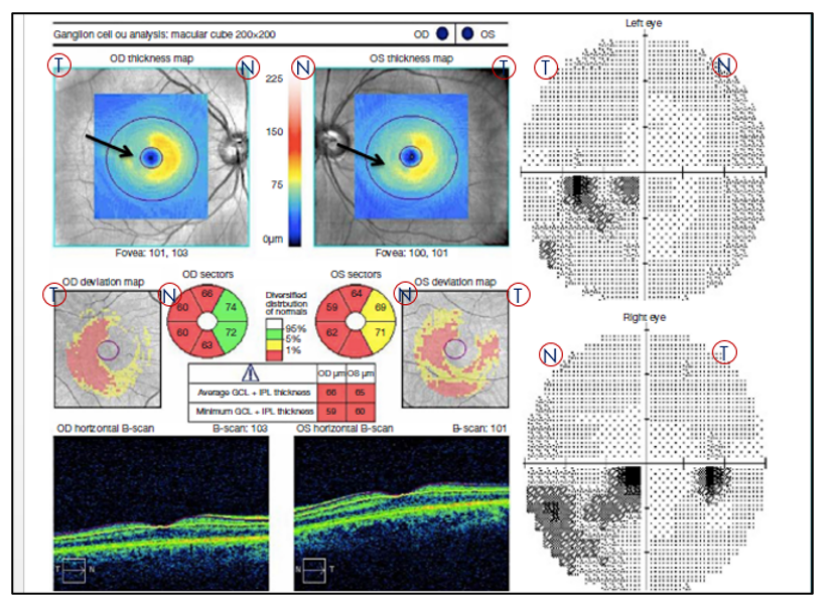

Figure 1: A 30-year-old woman with relapsing-remitting multiple sclerosis; (RRMS) presents with difficulty seeing the beginning of words caused by a left incongruous quandrantanopic visual field defect. The GCL analysis shows a pattern of right hemi-retinal loss (arrows) correlating to a right optic tract lesion [3].

\section{OCT detection of GCIPL thinning in non ON MS patients}

MS patients are known to have subclinical disease activity, which may be one reason why they harbour reduced OCT meas- 
ures of GCIP thickness, even in the absence of any past history of ON.

OCT GCIPL thickness can also be used to quantify changes in neuroaxonal integrity that may reflect retrograde degeneration from lesions anywhere along the course of the afferent visual pathway posterior to the optic nerve of MS patients [7].

\section{Non-arteritic ischemic anterior optic neuropathy (NAION)}

GCIPL thickness can detect axonal damage when the RNFL is swollen, Kupersmith demonstrated that at 1 month, only $10 \%$ eyes with NAION had RNFL thinning, while $76 \%$ had GCIPL thinning. GCIPL measurements correlate with visual field parameters, both in acute and chronic phase. Therefore, GCIPL measurements could be a biomarker of early structural loss in AION [9].

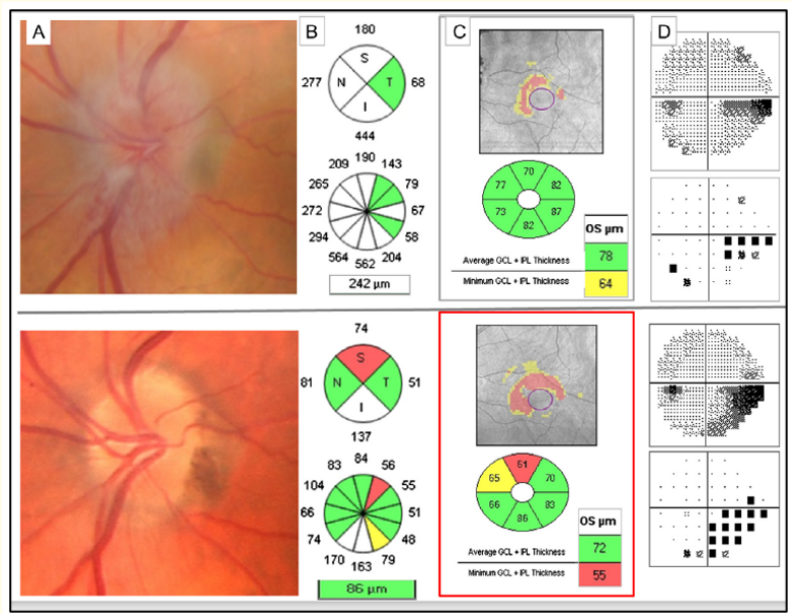

Figure 2: Left anterior ischemic optic neuropathy. Top row: One week after acute visual acuity loss. (A) Optic nerve swelling causes RNFL thickening that prevent evaluating if there is or not associated axonal loss (B). However, GCIPL analysis (C) shows an abnormal thinning that correlates with the inferior VF defect (D). Bottom row: two months after the acute episode. Superior optic disk pallor (A), superior RNFL quadrant thinning (B) and GCIPL thinning (C) with the corresponding inferior VF defect (D) [10].

\section{Leber hereditary optic neuropathy (LHON)}

It is an optic neuropathy secondary to mutations in mitochondrial DNA and affects young men. It is associated with retinal gan- glion cell degeneration (causing GCIPL thinning) and axonal loss (causing RNFL thinning) in the optic nerve (Figure 3) finally leading to optic atrophy (Figure 4) [11].

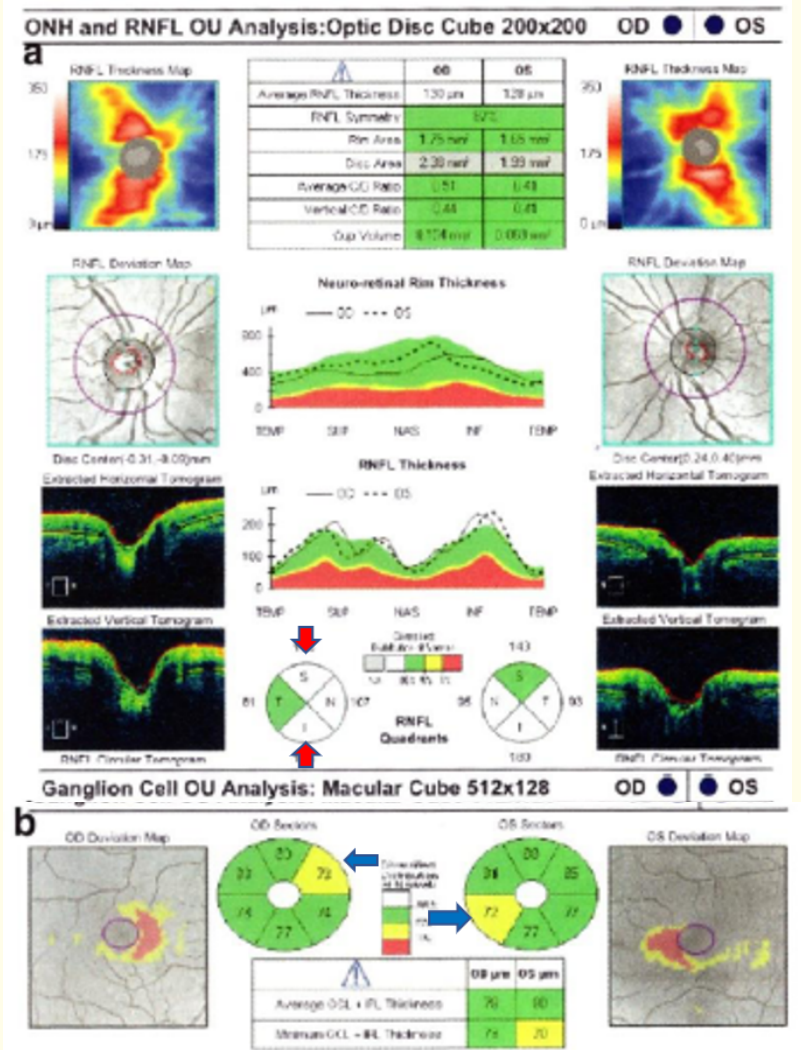

Figure 3: In the acute phase and in the first 6 months of onset, the RNFL analysis shows thickening in the upper and lower segments, (red arrows). The OCT analysis of macular thickness showed a thinning of the GCL (blue arrows) at the early stage in the sectors of the inner ring [12].

More studies will provide information about if early changes are present in carriers and in subacute or early LHNO cases [10].

\section{Compressive optical neuropathies}

Compressive optical neuropathies of the visual pathway can lead to severe impairment of visual function if they are discovered late. 

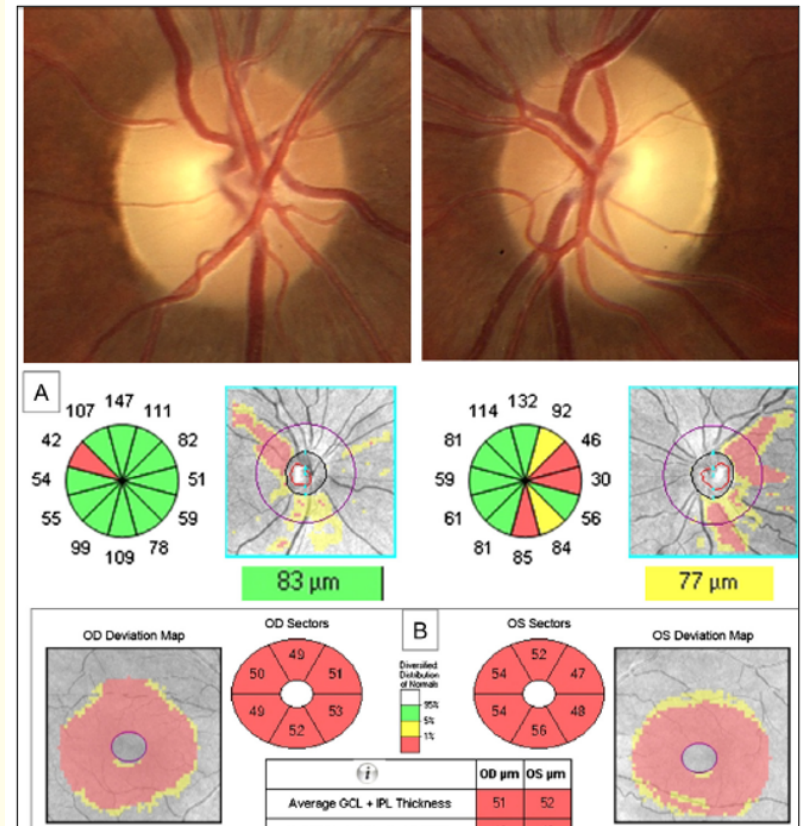

$83 \mu \mathrm{m}$

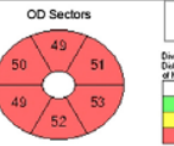

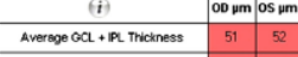
Mrimun $\mathrm{OCL}+\mathrm{PL}$ Thidiness

Figure 4: 24-Year-old male with Leber optic neuropathy. Fundus examination reveals a bilateral temporal pallor of the optic disk. GCIPL analysis (B) shows a bilateral severe thinning that is more striking than thinning yielded by RNFL analysis (A) [10].

OCT can be useful in the diagnosis, follow-up, and visual prognosis in this group of patients through quantification of the degree of axonal damage particularly by measuring the GCIPL thickness [12].

\section{Optic nerve meningioma}

- OCT GCIPL exam is important in predicting the visual prognosis: the less the GCIPL damage, the better is the visual prognosis

- After treatment, the group with normal GCIP experienced more pronounced improvement in VA, color vision and visual field, compared with the group with altered GCIPL [13].

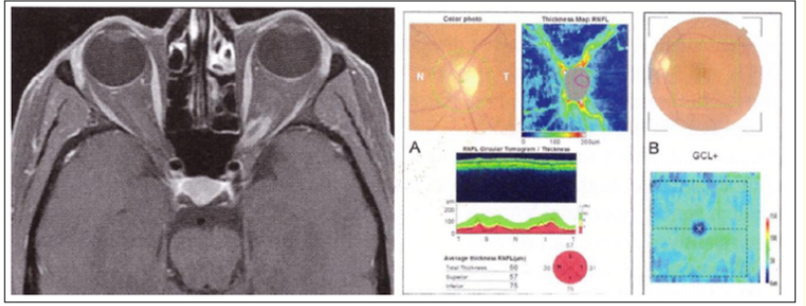

Figure 5: A, Thinning of the RNFL on OCT. B, Reduced GCL on OCT [13].

\section{Graves orbitopathy}

Graves Orbitopathy is an autoimmune inflammatory disorder that affects extraocular muscles and orbital fat, which are responsible for the dysthyroid optic neuropathy (Figure 6).

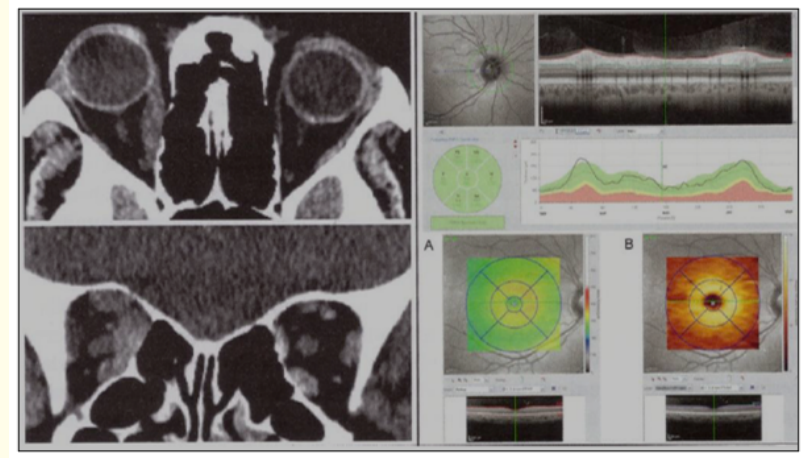

Figure 6: MRI shows enlarged medial and inferior rectus muscles. OCT shows normal peripapillary RNFL, normal macular full-thickness (A) and normal GCL (B). Expected good visual prognosis if treated at this stage [14].

Because prognosis is significantly improved when early diagnosis is made, patients with normal GCIPL measurements experienced an improvement in VA and visual field after decompression [15]. 


\section{Chiasmal lesions}

Chiasmal lesions may be caused by various etiologies, most common of which are pituitary adenoma, craniopharyngioma, meningioma, and aneurysm. Chiasmatic lesions can cause various degrees of damage to the GCIP layer that can be early detected by timely OCT measurements.

\section{Pituitary adenoma}

The pattern of macular GCIP loss is helpful in detecting some compressive optic neuropathies, by following a pattern similar to the visual field defect resulting from the compression.

An example is chiasmal injury, which causes binasal thinning of the GCIPL layer because the crossing nasal fibers are damaged with compression of the chiasm [3] (Figure 7).

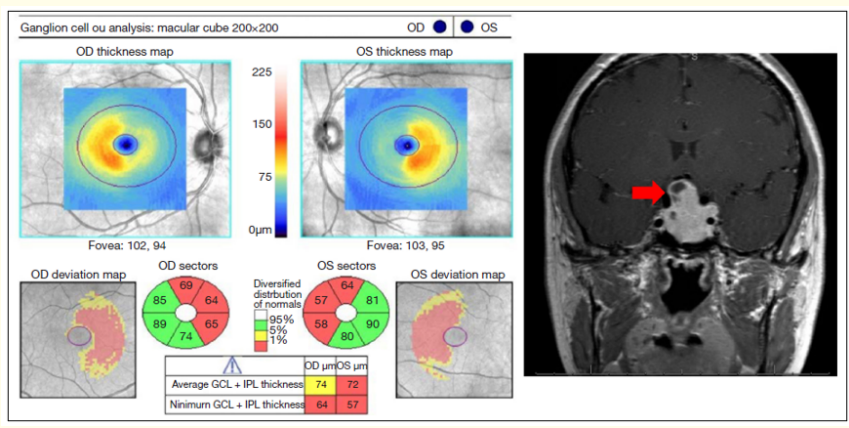

Figure 7: Pituitary macroadenoma causing chiasmal compression (red arrow). Binasal hemi-retinal GCIP thinning is seen with chiasmal compression [3].

Defining the pattern of GCIPL loss can be useful in differentiating chiasmal compression from other optic neuropathies, such as glaucoma for example, which tends to cause thinning of the macular GCIP that respects the horizontal meridian [16,17].

\section{Toxic optic neuropathies}

Toxic Optic Neuropathies occur due to either faulty ingestion of substances such as alcohol (methanol, ethylene glycol), or over dosage of anti-tuberculosis drugs (Ethambutol), antimalarial drugs, antiarrhythmic (digital, amiodarone), and antibiotics.
The patient usually presents with variable degrees of severity of painless, progressive, bilateral visual acuity decrease, dyschromatopsia, and central scotoma.

The pathogenesis is due to the damage of the papillomacular bundle, which is more vulnerable to damage due to the large unmyelinated segment and thin gauge [18].

Toxic optic neuropathy due to ethambutol

It usually becomes clinically manifested between 2 and 12 months after the onset of treatment. It is reversible if it is recognized early, although permanent toxic effects may occur at physiologic doses of 15 - $25 \mathrm{mg} / \mathrm{kg} /$ day. Ethambutol toxicity affects the visual pathway from the retinal ganglion cells, to the optic tracts [19].

GCIPL loss following ethambutol treatment, manifests before visual dysfunction and is more sensitive than p-RNFL thickness (Figure 8).

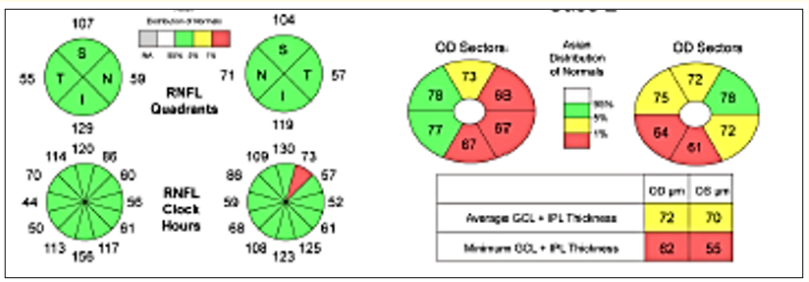

Figure 8: P-RNFL is normal in both eyes while GCIPL thickness was significantly lower than normal primarily in nasal sectors which is associated with the papillomacular bundles [20].

GCIPL measurements may as such indicate ethambutol withdrawal before serious visual impairment occurs [20].

\section{Neurodegenerative disorders}

GCIPL measurements are now being considered as a "window to the brain" and as a potential biomarker for neurodegenerative conditions.

GCIP thinning was found in patients with Alzheimer's disease, Parkinson's disease and frontotemporal dementia. 
More studies are necessary to assess whether GCIPL thickness represents an adjunctive biomarker for the diagnosis and followup of these pathologies [10].

\section{Conclusion}

OCT GCIPL measurement offers the opportunity to study neurological diseases in an objective and non-invasive manner. As mentioned above it aids in the diagnosis, treatment, and follow-up of optic neuropathies, in addition it can also have some prognostic value.

As OCT technology continues to advance, it will undoubtedly augment our ability to better understand the pathophysiology of optic neuropathies with consequent better diagnosis and management.

\section{Bibliography}

1. Costello F. "The afferent visual pathway: designing a structural-functional paradigm of multiple sclerosis". ISRN Neurology (2013): 134858.

2. Chen JJ and Kardon RH. "Avoiding Clinical Misinterpretation and Artifacts of Optical Coherence Tomography Analysis of the Optic Nerve, Retinal Nerve Fiber Layer, and Ganglion Cell Layer". Journal of Neuro-Ophthalmology 36 (2016): 417-438.

3. Chen JJ and Costello F. "The role of optical coherence tomography in neuro-ophthalmology (2018).

4. Chen JJ., et al. "Causes and Prognosis of Visual Acuity Loss at the Time of Initial Presentation in Idiopathic Intracranial Hypertension". Investigative Ophthalmology and Visual Science 56 (2015): 3850-3859.

5. Brandt AU., et al. "Monitoring the Course of MS With Optical Coherence Tomography". Current Treatment Options in Neurology 19 (2017): 15.

6. Bennett JL., et al. "Neuromyelitis optica and multiple sclerosis: Seeing differences through optical coherence tomography". Multiple Sclerosis Journal 21 (2015): 678-688.

7. Petzold A., et al. "Retinal layer segmentation in multiple sclerosis: a systematic review and meta-analysis". The Lancet Neurology 16 (2017): 797-812.
8. Thompson AJ., et al. "Diagnosis of multiple sclerosis: 2017 revisions of the McDonald criteria". The Lancet Neurology 17 (2018): 162-173.

9. Rebolleda G., et al. "Ganglion cell layer analysis unmasks axonal loss in anterior optic neuritis". Journal of Neuro-Ophthalmology 35.2 (2014): 165-167.

10. Rebolleda G., et al. "Neuro-ophthalmology Update OCT: New perspectives in neuro-ophthalmology". Saudi Journal of Ophthalmology 29 (2015): 9-25.

11. Eugenia IR. "The role of Optical Coherence Tomography in optic neuropathies". Romanian Journal of Ophthalmology 62.1 (2018): 3-14.

12. Barboni P., et al. "Retinal nerve fiber layer evaluation by optical coherence tomography in Leber's hereditary optic neuropathy". Ophthalmology 112 (2005): 120-126.

13. Loo JL., et al. "Use of optical coherence tomography in predicting post-treatment visual outcome in anterior visual pathway meningiomas". British Journal of Ophthalmology 97.11 (2013): 1455-1458.

14. Grzybowski A and Barboni P. "OCT in central nervous system disease. The eye as a window to the brain, 2016, Switzerland, Springer International Publishing (2016): 73.

15. Forte R., et al. "Evaluation of retinal nerve fiber layer with optic nerve tracking optical coherence tomography in tyroid-associated orbitopathy". Ophtalmologica 224.2 (2010): 116-121.

16. Tieger MG., et al. "Ganglion Cell Complex Loss in Chiasmal Compression by Brain Tumors". Journal of Neuro-Ophthalmology 37 (2017): 7-12.

17. Yum HR., et al. "Macular Ganglion Cell Analysis Determined by Cirrus HD Optical Coherence Tomography for Early Detecting Chiasmal Compression". PLoS One 11 (2016): e0153064.

18. Wang MY and Sadun AA. "Drug-related mitochondrial optic neuropathies". Journal of Neuro-Ophthalmology 33 (2013): 172-178.

19. Kinoshita J., et al. "Retinal function and morphology in monkeys with ethambutol-induced optic neuropathy". Investigative Ophthalmology and Visual Science 53 (2012): 7052-7062. 
20. Sheng., et al. "BMC Ophthalmology. Analysis of structural injury patterns in peripapillary retinal nerve fibre layer and retinal ganglion cell layer in ethambutol induced optic neuropathy 21 (2021): 132.

Volume 4 Issue 11 November 2021

C) All rights are reserved by Ahmed Darwish. 